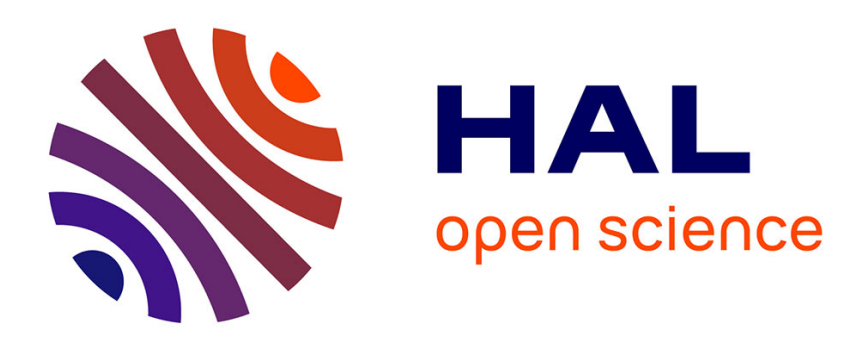

\title{
Experimental and Numerical Investigation of Void Nucleation in an AlMgSi Alloy
}

\author{
O. Søvik
}

\section{To cite this version:}

O. Søvik. Experimental and Numerical Investigation of Void Nucleation in an AlMgSi Alloy. Journal de Physique IV Proceedings, 1996, 06 (C6), pp.C6-155-C6-164. 10.1051/jp4:1996615 . jpa-00254443

\section{HAL Id: jpa-00254443 https://hal.science/jpa-00254443}

Submitted on 1 Jan 1996

HAL is a multi-disciplinary open access archive for the deposit and dissemination of scientific research documents, whether they are published or not. The documents may come from teaching and research institutions in France or abroad, or from public or private research centers.
L'archive ouverte pluridisciplinaire HAL, est destinée au dépôt et à la diffusion de documents scientifiques de niveau recherche, publiés ou non, émanant des établissements d'enseignement et de recherche français ou étrangers, des laboratoires publics ou privés. 


\title{
Experimental and Numerical Investigation of Void Nucleation in an AlMgSi Alloy
}

O.P. Søvik

Raufoss Hydro Automotive Research Centre, P.O. Box 41, 2831 Raufoss, Norway

\begin{abstract}
The nucleation of microvoids from second phase particles in an AlMgSi alloy has been investigated. The work has comprised detailed stress analyses of elastic particles situated in an elastic-plastic matrix as well as damage mechanics analyses of the ductile fracture behaviour of smooth and notched axisymmetric tensile specimens. Based on the micromechanical analyses of isolated particles, general expressions relating local stresses in the particles to the stresses on a mesoscopic (continuum) level have been derived. These relationships have been incorporated into the classical Gurson model. Combined with a failure criterion based on a physical coalescence mechanism, a calibration with experimental results enabled the establishment of a set of micromechanical parameters which are transferable between different stress triaxialities.
\end{abstract}

\section{Introduction}

During the last years, the constitutive model introduced by Gurson [5] has been used extensively for simulating ductile damage. Despite the many simplifications adopted by the model, numerous investigations have demonstrated its capability to predict ductility limits of engineering materials and structures.

The successful application of the Gurson model relies upon an appropriate selection of the micromechanical parameters included in the model. A direct determination of these parameters based on metallographical examinations is however difficult since there appears to be no unique relation between the micromechanical parameters and the actual microstructural characteristics of the material. A more commonly applied procedure for determining the micromechanical parameters is based on a dual approach involving experimental testing as well as FE analyses of axisymmetric tensile specimens (see e.g. [12]). The FE analyses calculate the macroscopic response of the specimens using the constitutive equations of the Gurson model. The choice of nucleation parameters (and the Tvergaard parameters $q_{1}$ and $q_{2}$ ) is either arbitrary or based on metallographical examinations. The final step is to compare the experimentally observed response with the numerical calculations and then adjust the coalescence parameters so that the fulfilment of the chosen coalescence criterion coincides with failure in the tested specimens.

A frequently observed problem with the Gurson model is the lack of transferability between different stress states. A set of micromechanical parameters determined for one particular stress state does not necessarily apply to other stress states. In a recent investigation by the author [8], the effect of void shape on the transferability of micromechanical parameters was studied. The void shape has previously been shown to have a significant effect on damage evolution $[9,10]$. This effect depends on stress triaxiality, and it was therefore expected to also be of significance for the transferability problem. Although it was demonstrated that void shape effects may play a significant role, it was concluded that these effects cannot alone be responsible for the observed lack of transferability. 


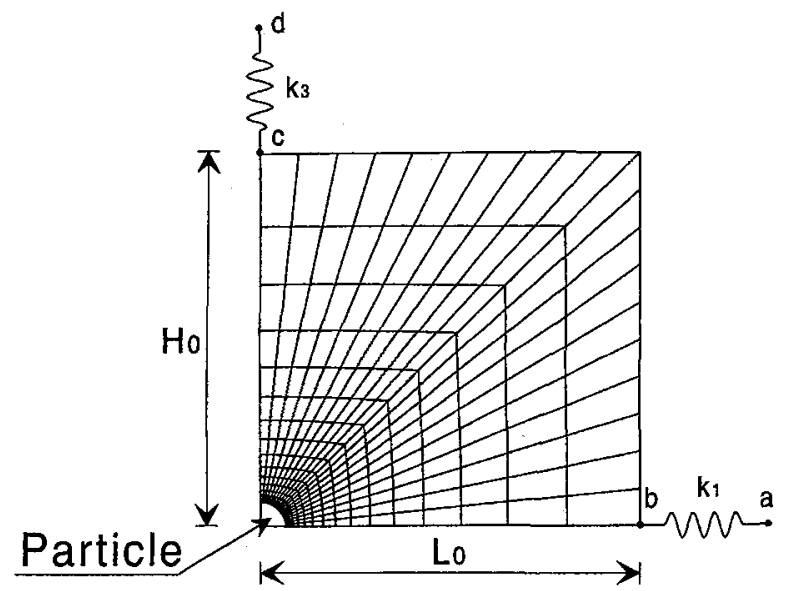

\section{Particle}

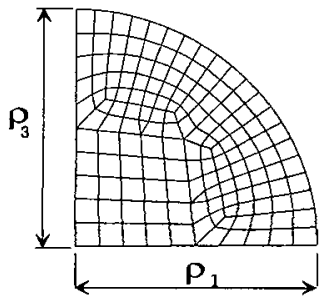

Figure 1: Axisymmetric FE model of material unit cell containing an isolated elastic particle

In the present work, another possible source of the stress state dependence of micromechanical parameters is investigated; namely the nucleation model applied. In current applications of the Gurson model, it is frequently assumed that the voids are either initially present or that they are nucleated upon the attainment of a certain nucleation strain. The latter may be a reasonable assumption in the case of very small particles $(<1 \mu \mathrm{m})$ since the local stresses then are mainly determined by the accomodation of plastic strains around the particles. For larger particles, however, many investigations (e.g. $[1,3]$ ) have indicated that the stresses at the particles are determined by the global stress state. In such cases, a nucleation model based on a constant nucleation strain may be inappropriate. The main aim of the present work is to investigate whether the adoption of a stress based nucleation model will improve the stress state transferability of micromechanical parameters for the Gurson model.

In the first part of this paper, the main results of some micromechanical stress analyses of elastic particles surrounded by an elastic-plastic matrix will be given. In the second part, the results from the micromechanical analyses will be used in connection with the Gurson model in an attempt to establish micromechanical parameters that are transferable between different stress states.

\section{Micromechanical stress analyses}

In order to evaluate the local stresses around an elastic particle situated in an elastic-plastic matrix, an axisymmetric finite element (FE) model of a representative material element (unit cell) was established. The FE mesh is shown in Figure 1. The spring elements are introduced in order to control the stress triaxiality. The FE model is described in more detail in $[9,10]$.

In consistency with a common damage mechanics approach, it is convenient to distinguish between local and global quantities. In the present work, local quantities within the unit cell will be referred to as micro-quantities. Averaged, or global quantities will be denoted meso-quantities. With this notation, the term macroscopic should only be used to describe an assembly of unit cells (e.g. a structure or a component). 


\subsection{Mesoscopic stresses and strains}

The mesoscopic stress components are defined in terms of the net forces acting at the cell edges in the following way

$$
\Sigma_{11}=\frac{F_{1}}{4 \pi\left(L_{0}+u_{1}\right)\left(H_{0}+u_{3}\right)}, \quad \Sigma_{33}=\frac{F_{3}}{\pi\left(L_{0}+u_{1}\right)^{2}}
$$

where $F_{1}$ and $F_{3}$ are the concentrated loads applied in the radial and axial direction, respectively. The former is assumed to be the integral of a force distributed along the circumference.

Due to the axisymmetry, $\Sigma_{11}=\Sigma_{22}$ and $\Sigma_{33}$ are principal stresses and the mesoscopic effective and hydrostatic stresses can thus be expressed

$$
\Sigma_{e}=\left|\Sigma_{33}-\Sigma_{1 I}\right|, \quad \Sigma_{h}=\frac{1}{3}\left(\Sigma_{33}+2 \Sigma_{11}\right)
$$

The mesoscopic stress triaxiality factor is defined as

$$
T=\frac{\Sigma_{h}}{\Sigma_{e}}=\frac{1}{3}\left(\frac{1+2 \theta}{|1-\theta|}\right)
$$

where $\theta=\Sigma_{11} / \Sigma_{33}$ is the stress proportionality factor.

The mesoscopic strains are defined as logarithmic strains in the following manner

$$
\eta_{11}=\int_{L_{0}}^{L} \frac{d L}{L}=\ln \left(\frac{L_{0}+u_{1}}{L_{0}}\right), \quad \eta_{33}=\int_{H_{0}}^{H} \frac{d H}{H}=\ln \left(\frac{H_{0}+u_{3}}{H_{0}}\right)
$$

The corresponding mesoscopic effective strain is conveniently defined

$$
\eta_{e}=\frac{2}{3}\left|\eta_{33}-\eta_{11}\right|
$$

\subsection{Material descriptions}

The matrix material is assumed to obey a uniaxial stress-strain relation of the following form:

$$
\bar{\sigma}(\bar{\epsilon})= \begin{cases}E \bar{\epsilon} & ; \bar{\epsilon} \leq \frac{\sigma_{0}}{E} \\ \sigma_{0}\left(\frac{E \bar{\epsilon}}{\sigma_{0}}\right)^{n} & ; \bar{\epsilon}>\frac{\sigma_{0}}{E}\end{cases}
$$

where $\bar{\sigma}$ and $\bar{\epsilon}$ are the uniaxial stress and strain, respectively, $\sigma_{0}$ is the uniaxial yield stress, $E$ is Young's modulus and $n$ is the hardening exponent. In the analyses reported here, Young's modulus was set to $70 \mathrm{GPa}$, the yield stress to $260 \mathrm{MPa}$ and the hardening exponent to 0.1 .

The particle was specified as purely elastic with a Young's modulus of $130 \mathrm{GPa}$ and a Poisson's ratio of 0.33 .

\subsection{Stresses at the particle-matrix interface}

The interactions between the matrix and particle were modelled using the special contact formulation in ABAQUS [6]. In this approach, the contact problem is defined in terms of two surfaces, of which one is denoted the master and the other the slave. If the two surfaces are brought into contact, ABAQUS will automatically introduce the constraints required for forcing the nodes on the slave surface to remain on the master surface as long as the contact conditions prevail. In the present work, the contact was specified as permanently bonded. The advantage of using this procedure is that the stresses at the interface are automatically calculated and given with components normal to and tangential to the interface, respectively.

Figure 2a) shows the variation of normal stresses along the interface between a spherical 


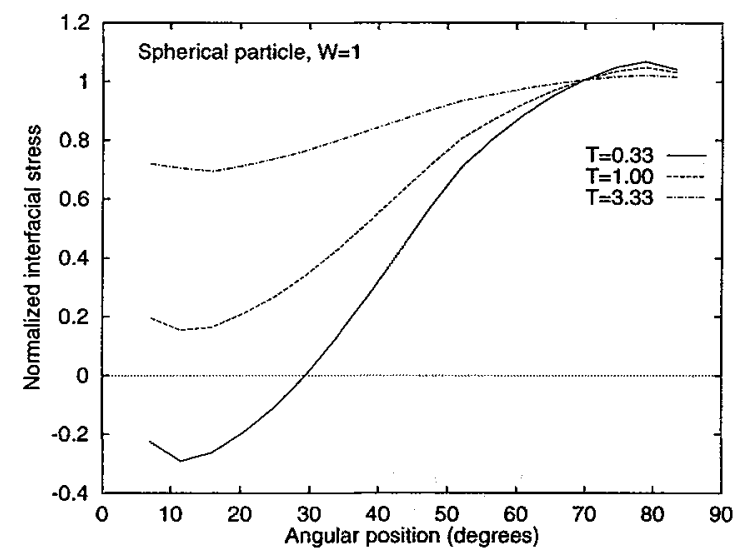

a)

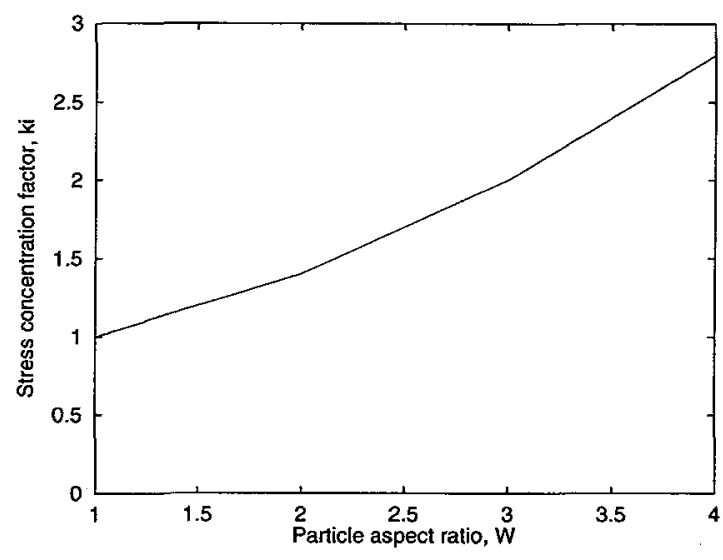

b)

Figure 2: a) Variation of normal stresses at the interface of a spherical particle and matrix. The effective mesoscopic strain is close to $5 \% \mathrm{~b}$ ) estimated values of $k_{i}$ for different particle shapes

particle and the matrix. The angular position is referred to the radial direction, i.e. an angular position of $90^{\circ}$ corresponds to the pole of the particle. The normal stresses are normalised with respect to Argon's [1] estimate of the maximum interfacial normal stress

$$
\sigma_{i}^{\max }=k_{i} \Sigma_{e}+\Sigma_{h}
$$

where $k_{i}$ is a constant depending on the particle shape.

The maximum normalised stress is close to unity for all the stress states studied and it remains so over a relatively wide range of deformation. The calculations therefore lend credibility to the Argon model.

Similar calculations as those depicted in Figure 2 a) were performed for particles with aspect ratios $W\left(=\rho_{3} / \rho_{1}\right)$ ranging from one to four. It was found that with an appropriate adjustment of the $k_{i}$-factor, the Argon model reasonably well predicts the maximum normal stress over the interface. However, for the spheroidal particles, the normalised stresses tended to vary more with the strain level than was the case for the spherical particle. Figure $2 \mathrm{~b}$ ) shows the estimated value of the $k_{i}$-factor as a function of particle shape.

\subsection{Stresses in the particle cross-section}

Figure 3 shows the distribution of normal stresses $\left(\sigma_{33}\right)$ across the cross-section of particles with aspect ratios ranging from 1 to 4 . The stresses are relatively constant over the cross-section, but the stress level increases markedly with increasing aspect ratio. The stress raising effect of a superimposed hydrostatic stress is also apparent.

The cross-sectional normal stress in the centre of the particle was found to be conveniently approximated by the following expression

$$
\sigma_{c}^{\max }=k_{c} \Sigma_{e}+\Sigma_{h}
$$

where $k_{c}$ is a constant. For all the calculations reported here, the $k_{c}$-factor was found to be approximately 1.25 times $k_{i}$ as given in Figure $2 \mathrm{~b}$ ).

Figure $3 \mathrm{~b}$ ) shows the variation of the cross-sectional normal stress with effective strain. The stresses are normalised with those predicted by equation (8). As can be seen, the normalised stresses 


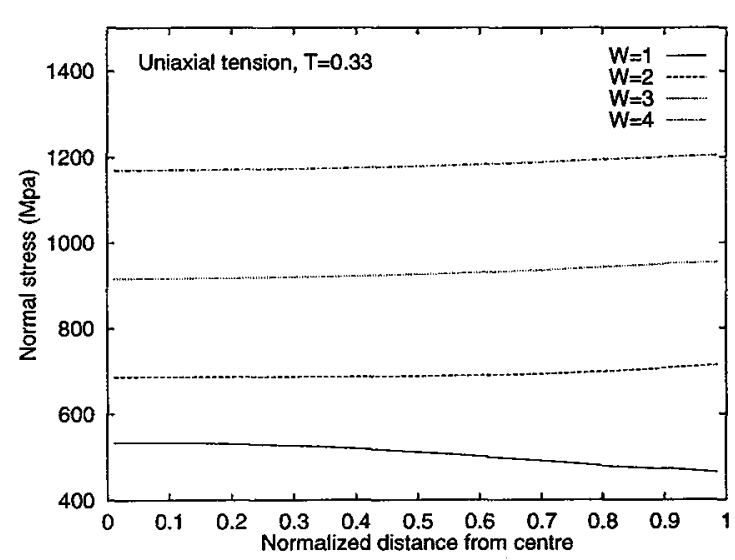

a)

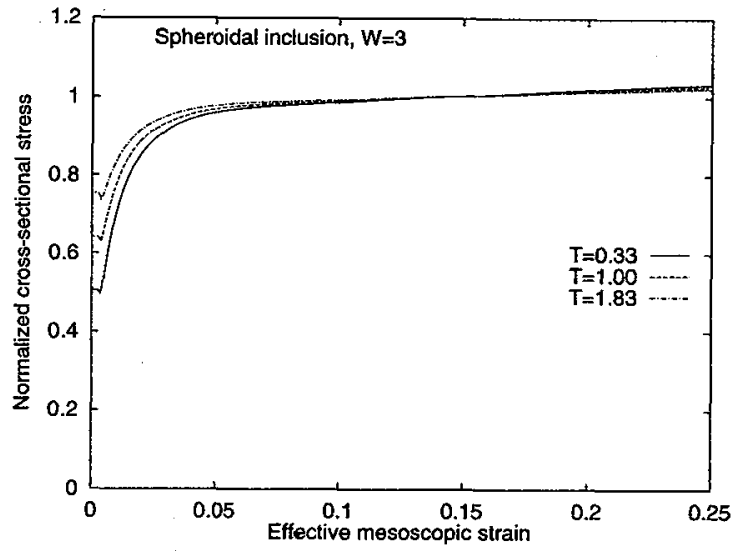

b)

Figure 3: a) Typical variation of normal stresses over the cross-section of particles with varying aspect ratio, and b) typical variations of normalised cross-sectional stress with effective strain for a spheroidal particle with aspect ratio equal to three.

stay close to unity over a wide range of both strain and stress triaxiality. Similar results were found also for other particle shapes, thus lending confidence to the expression in equation (8).

Experimental investigations of void nucleation from second-phase particles and inclusions often show an increased tendency for particle fracture with increasing particle aspect ratio (e.g. [2]). By using equations (8) and (7), the ratio of maximum cross-sectional stress to maximum particlematrix interfacial stress can be evaluated

$$
\frac{\sigma_{c}^{\max }}{\sigma_{i}^{\max }}=\frac{k_{c} \Sigma_{e}+\Sigma_{h}}{k_{i} \Sigma_{e}+\Sigma_{h}}=\frac{1.25 k_{i}+T}{k_{i}+T}
$$

Although the ratio increases with increasing particle aspect ratio for all stress triaxialities, the increase is too small to be any major explanation to the abovementioned observations.

\section{Simulation of ductile fracture in axisymmetric tensile specimens}

In order to investigate the effects of using a stress controlled nucleation model, detailed simulations of smooth and notched axisymmetric tensile specimens were performed. Similar specimens of an AlMgSi alloy have previously been tested experimentally, thus allowing for a determination of micromechanical parameters for this alloy.

It has been shown [11] that the nucleation phase in aluminium alloys may constitute the major part of the total ductility. An accurate prediction of the nucleation instant is therefore an essential step in any simulation of ductile fracture in these alloys.

\subsection{Experimental testing}

\subsubsection{Material description}

The test material was taken from an extruded AlMgSi alloy. Chemical composition is shown in table 1. Metallographical investigations [7] revealed that the only constituent particles were AlFeSi. These particles were oriented with their longitudinal axes in the direction of extrusion and had an average aspect ratio of about 2.7 . 


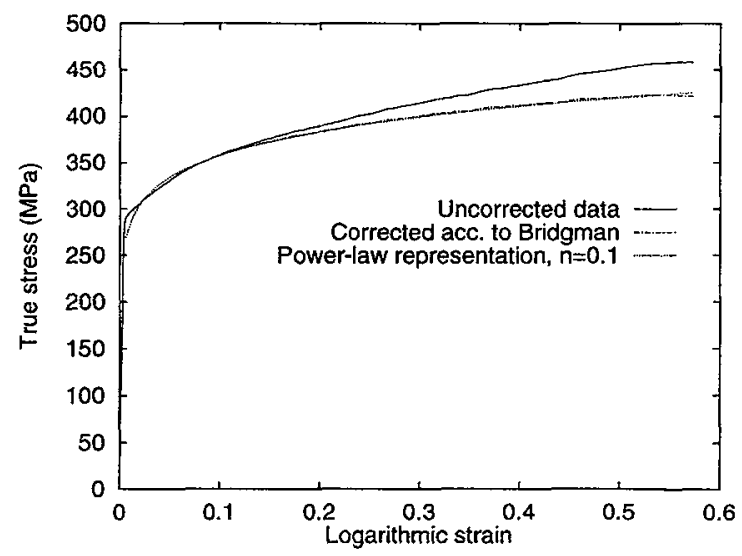

Figure 4: True stress-strain curve for the studied aluminium alloy

\subsubsection{Mechanical behaviour}

In addition to smooth specimens (TSS), notched specimens with three different notch radii were extracted in the longitudinal direction (i.e. the direction of extrusion). The notch radii applied were 2 (TSR2), 0.8 (TSR08) and $0.4 \mathrm{~mm}$ (TSR04). During the tensile testing (at room temperature), the applied load and the contraction were recorded. The diameter reduction was measured by means of a special device consisting of four inductive displacement gauges placed symmetrically around the circumference. The values reported here were obtained as average displacements from these four gauges.

Three specimens of each geometry were tested but the variation in mechanical response among the parallels was negligible. The smooth specimens were also provided with an extensiometer for more accurate recordings of displacements. These recordings were used for establishing a 'true' stress-strain curve, see Figure 4.

\subsection{Numerical calculations}

The Gurson model was implemented through the UMAT user subroutine in ABAQUS. The integration of the rate equations is based on a generalised mid-point algorithm developed by Zhang [14].

\subsubsection{Nucleation model}

Metallographic examinations of the tested aluminium alloy showed that the voids were formed exclusively by particle fracture. It was therefore assumed that the maximum stress in the particle cross-section is the controlling factor for initiation. Assuming this maximum stress to be given by equation (8), the corresponding nucleation criterion can be expressed

$$
k_{c} \Sigma_{e}+\Sigma_{h} \geq \sigma_{c}^{c r i t}
$$

where $\sigma_{c}^{\text {crit }}$ is the fracture strength of the particle.

\begin{tabular}{||c|c|c|c|c||}
\hline \hline $\mathrm{Si}$ & $\mathrm{Mg}$ & $\mathrm{Mn}$ & $\mathrm{Cr}$ & $\mathrm{Fe}$ \\
\hline 0.7 & 0.57 & 0.24 & 0.12 & 0.23 \\
\hline
\end{tabular}

Table 1: Chemical composition (wt\%) of the studied aluminium alloy 
In current applications of the Gurson model, the increase in porosity due to nucleation of new voids is often assumed to have the following form:

$$
\dot{f}_{n u c l e a t i o n}=A \dot{\sigma}_{M}+B \dot{\Sigma}_{k k} / 3
$$

where $A$ and $B$ represent nucleation intensities.

It is seen that the nucleation criterion in equation (10) can be accomplished through the general formula in equation (11) if $A=k_{c} B$ and the matrix flow stress $\sigma_{M}$ is assumed to be approximately equal to the mesoscopic flow stress. The latter is a good approximation if the material has a dilute concentration of voids.

Using a purely stress-controlled nucletion model may lead to some numerical difficulties. These are mainly attributable to the general softening effect of cavitation, i.e. a (positive) increment in porosity may give a negative increment in effective stress. From a numerical point of view, it is more convenient to deal with effective plastic strain since this variable will always be a monotonically increasing function of time. In the present work it was therefore decided to adopt a dual nucleation model. The nucleation process itself is strain controlled (i.e. $B=0$ ), whereas the nucleation strain will depend on the stress state. Following an approach introduced by Chu and Needleman [4], the nucleation intensity $A$ is defined by means of a normal distribution with mean value $\epsilon_{N}$ and standard deviation $S_{N}$

$$
A=\frac{k_{c} f_{N}}{S_{N} \sqrt{2 \pi}} \exp \left\{-\frac{1}{2}\left[\frac{\bar{\epsilon}^{p}-\epsilon_{N}}{S_{N}}\right]^{2}\right\}
$$

where $f_{N}$ is a scaling factor that can be regarded as the total amount of particles that are available for nucleation.

The nucleation strain $\epsilon_{N}$ is computed separately for each point in the specimens according to the actual stress state. In order to include the lower 'half' of the normal distribution in equation (12), it is necessary to know the nucleation strain before the analysis actually reaches that strain. The estimation of nucleation strain was accomplished in the following manner: At the beginning of each increment, the matrix flow stress corresponding to a 'trial' strain equal to the current plastic strain plus $3 S_{N}$ is determined. This flow stress and the current mesoscopic hydrostatic stress are then entered into the nucleation criterion in equation (10). If the criterion is met, the nucleation strain for that point is set equal to the trial strain. The nucleation strain, once determined for a point, will remain unchanged throughout the rest of the deformation history. This procedure assumes that $\sigma_{M} \approx \Sigma_{e}$ and that $\Sigma_{h}$ does not change significantly during a plastic strain increment of $3 S_{N}$.

\subsubsection{Void growth}

After nucleation the voids will probably have a markedly oblate shape. According to the results in [10], such voids will grow significantly faster than spherical voids of the same volume fraction. However, the nucleation process as well as the early stages of void growth are very complex. Large parts of the broken particles may remain bonded to the matrix and probably alter the growth pattern. Since these aspects are not known, it was decided not to include the influence of void shape in the present investigation.

\subsubsection{Void coalescence}

As mentioned above, the most commonly used procedure for determining micromechanical parameters for the Gurson model is to choose a set of nucleation parameters and then adjust the coalescence parameter (normally the critical void volume fraction, $f_{c}$ ) until the computations fit the experimental results.

In the present work, an approach introduced by Zhang [15] was adopted. This approach is based on an introduction of Thomason's coalescence model [13] into the Gurson model. Further details of this implementation is given in [9]. 
By letting the void coalescence be a 'natural' consequence of the damage evolution, the calibration of micromechanical parameters can be based on the nucleation parameters rather than the coalescence parameters. In this way it is believed that more 'realistic' parameters can be established and thereby improve the chances of obtaining parameters that are stress state independent.

\subsubsection{Results}

Figure 5 shows an example of the results obtained in the simulations. These results were obtained with a nucleation stress $\sigma_{N}$ of $1100 \mathrm{MPa}$. The volume fraction of void generating particles $f_{N}$ was specified as $1 \%$. This value is close to the area fraction of AlFeSi particles found in the metallographical analyses, and should therefore be a realistic value. The standard deviation $S_{N}$ (cfr. equation (12)) was set to $1 \%$, i.e. approximately all particles have fractured during a strain interval of about $6 \%$. The $k_{c}$-factor was set to 2.25 which corresponds to the average particle aspect ratio in the investigated alloy.

Figure 5a) shows the numerically obtained load vs. diameter reduction curves together with those found experimentally. Apart from the smooth specimen (TSS), the numerical predictions follow the experiments quite well both with respect to load level and final fracture. For the smooth specimens, the numerical simulations overpredict the falure strain. One reason for the observed discrepancy is beleived to be the mechanical anisotropy in the specimens. During the experimental testing, the three smooth specimens exhibited a very asymmetric deformation, i.e. the necking section developed into an ovale shape. It is therefore not unexpected that this specimen geometry gives the poorest fit between experiments and simulations. For the notched specimens, the deviation from an axisymmetric deformation mode appeared to be significantly less than that of the smooth specimens.

Figure 5b) shows the evolution of damage in the points where coalescence first occurred. For the smooth specimen and the two geometries with the largest notch radii, coalescence occurred close to the centre of the specimens. For specimen TSR04, however, coalescence occured first ahead of the notch root. There is therefore a shift in initial coalescence location from the centre of the specimen to the notch root as the notch root radius increases. This shift was observed already for specimen TSR08 where the first coalescence occurred about simultaneously over a large part of the central region.

As could be expected, the incipient void nucleation starts at a much higher strain level for the smooth specimen than for the three other specimen geometries. This is a direct consequence of the lower triaxiality in the smooth specimen. Looking at Figure 5, one may be led to the conclusion that the same results could have been obtained with a constant nucleation strain of about $5 \%$. However, this is not the case. With a constant nucleation strain, the damage distribution would have been significantly altered. For specimen TSR08, for instance, final failure would have been predicted at a much earlier stage because the most severely damaged region would then have been in the highly strained (but with relatively low stress triaxiality) region in front of the notch root.

\section{Conclusions}

Micromechanical stress analyses have demonstrated that both the maximum normal stress at the particle-matrix interface and the maximum cross-sectional stress in axisymmetric particles can be evaluated in terms of mesoscopic quantities.

Numerical simulations of the ductile fracture behaviour of smooth and notched tensile specimens have shown that the applied nucleation model may have a significant effect on the transferabiblity of micromechanical parameters for the Gurson model.

With an appropriate choice of micromechanical parameters, the Gurson model gives good predictions of ductility for a wide range of stress states. An appropriate nucleation model in combination with a micromichanical based coalescence criterion, shows good prospects with respect to the ability to determine physically realistic nucleation parameters. 


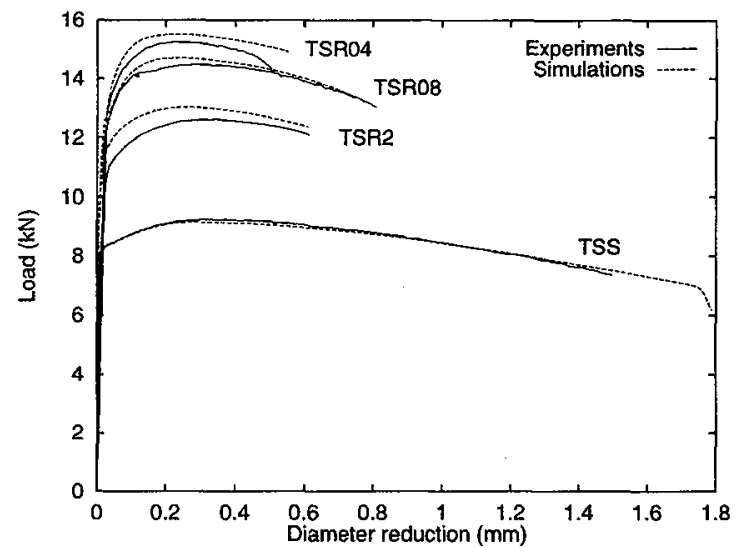

a)

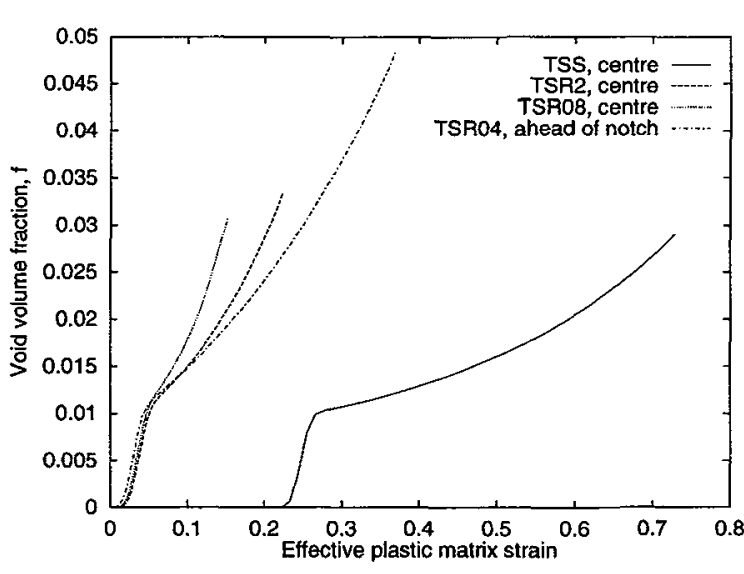

b)

Figure 5: a) Experimental (solid lines) and simulated (dotted lines) load vs. diameter reduction curves, and $b$ ) evolution of damage in the points where coalescence first occured.

\section{Acknowledgements}

The present work has received support from The Research Council of Norway (Programme for Supercomputing) through a grant of computing time. Many stimulating discussions with Dr. Z. Zhang at SINTEF Materials Technology are also highly appreciated.

\section{References}

[1] A.S. Argon. Formation of cavities from nondeformable second-phase particles in low-temperature ductile fracture. Journal of Engineering Materials and Technology, Trans. ASME, 98:60-68, 1976.

[2] A.S. Argon, J. Im, and R. Safoglu. Cavity formation from inclusions in ductile fracture. Metallurgical Transactions A, 6A:825-837, 1975.

[3] F.M. Beremin. Cavity formation from inclusions in ductile fracture of A508 steel. Metallurgical Transactions A, 12A:723-731, 1981.

[4] C.C. Chu and A. Needleman. Void nucleation effects in biaxially stretched sheets. Journal of Engineering Materials and Technology, 102:249-256, 1980.

[5] A.L. Gurson. Continuum theory of ductile rupture by void nucleation and growth: Part I Yield criteria and flow rules for porous ductile media. Journal of Engineering Materials and Technology, pages 2-15, Jan 1977.

[6] Hibbit, Carlson \& Sorensen, Inc. ABAQUS Version 5.5, User's manual, 1995.

[7] J.H. Lange. Damage mechanics evaluation of aluminium weldments with the Gurson model. Testing, metallographic examination and FE-calculations. Master's thesis, Dept. of Machine Design and Material Technology, Norwegian University of Science and Technology, Trondheim, Norway, 1994.

[8] O.P. Søvik. The effect of non-spherical void shape on the evolution of ductile damage. In $11^{\prime}$ th European Conference on Fracture, Poitiers-Futuroscope, France, September 1996. 
[9] O.P. Søvik. Numerical modelling of ductile fracture - A damage mechanics approach. $\mathrm{PhD}$ thesis, Norwegian University of Science and Technology, Trondheim, Norway, 1996.

[10] O.P. Søvik and C. Thaulow. Growth of spheroidal voids in elastic-plastic solids. Accepted for publication in Fatigue and Fracture of Engineering Materials and Structures.

[11] H. Van Stone, T.B. Cox, and J.R. Crow Jr. Microstructural aspects of fracture by dimpled rupture. International Metals Reviews, 30, 1985.

[12] D.-Z. Sun, R. Kienzler, B. Voss, and W. Schmitt. Application of micromechanical models to the prediction of ductile fracture. In S.N. Atluri et al., editor, Fracture Mechanics: TwentySecond Symposium (Volume II), ASTM STP 1131, pages 368-378, Philadelphia, 1992. American Society for Testing and Materials.

[13] P.F. Thomason. Three-dimensional models for the plastic limit-loads at incipient failure of the intervoid matrix in ductile porous solids. Acta Metallurgica, 33(6):1079-1085, 1985.

[14] Z.L. Zhang and E. Niemi. A class of generalized mid-point algorithms for the Gurson-Tvergaard material model. International Journal of Numerical Methods in Engineering, 38:2033-2053, 1995.

[15] Z.L. Zhang and E. Niemi. A new failure criterion for the gurson-tvergaard dilational constitutive model. International Journal of Fracture, 70:321-334, 1995. 